\title{
Neuropathic pain in chronic idiopathic axonal neuropathy: Fast pain reduction after topical phenytoin cream
}

\author{
*Corresponding Author(s): Jan M keppel Hesselink \\ Faculty of health, University of Witten-Herdecke, \\ Germany \\ Institute for Neuropathic Pain, The Netherlands \\ Email: jan@neuropathie.nu
}

Received: Aug 31, 2018

Accepted: Nov 12, 2018

Published Online: Nov 13, 2018

Journal: Journal of Case Reports and Medical Images

Publisher: MedDocs Publishers LLC

Online edition: http://meddocsonline.org/

Copyright: (C) keppel Hesselink JM (2018). This Article is distributed under the terms of Creative Commons Attribution 4.0 International License

Keywords: CIAP; Cryptogenic; Neuropathy; Idiopathic; Topical; Treatment

\section{Introduction}

Chronic idiopathic axonal neuropathy is a progressive sensory or sensorimotor polyneuropathy that affects elderly patients. Sometimes this neuropathy is referred to as cryptogenic neuropathy. These patients often suffer from peripheral neuropathic pain. Clinical studies evaluating analgesic therapy in this population however are missing [1].

Since 2010 we regularly see these patients in our institute for neuropathic pain in the Netherlands, and one of the treatments we evaluated in the past was the supplement palmitoylethanolamide, $1200 \mathrm{mg} /$ day, dosed in 3 times daily in capsules of 400 $\mathrm{mg}$. This supplement often reduces CIAP pain after some weeks,

\begin{abstract}
Pain in Chronic Idiopathic Axonal Neuropathy (CIAP) is often treated based on guidelines, not specifically developed for CIAP patients, and we can only extrapolate findings from for instance Painful Diabetic Neuropathy (PDN) to our CIAP patients. Clearly, the pathogenesis and pathophysiology of CIAP will be different from that of most PDN patients. Many CIAP patients complain of the intolerable side-effects of current analgesic medication. In order to help those patients, we developed a treatment-regime consisting of the supplement palmitoylethanolamide and topical formulations of analgesics. This combination is rarely complicated by side-effects. One of our most recent topical formulation we developed contains $10 \%$ phenytoin, a broad acting sodium-channel blocker. It can also be used as a stand-alone therapy as we will discuss.
\end{abstract}

We present a case of a patient, diagnosed as CIAP, treated successfully by the cream, reporting a quick onset of action; at a YouTube, you can listen to the impression of the patient himself, in order to add the patient's story to our case description. but of course there will always be non-responders due to reasons which are unclear, but most probably related to the pathogenesis of the pain [2]. For patients that are non-responders we developed a topical pain cream, containing the old co-analgesic compound phenytoin [3]. Meanwhile we discovered, that the cream can be easily used as a stand-alone therapy [3].

In CIAP patients suffering from pain, pain characteristics are mostly burning, suggestive for the involvement of a small fiber component [4]. Most patients explain they feel the burning pain in the skin, this was for us a pointer to develop topical formulations containing analgesics. In patients suffering from small 
fiber neuropathy and pain we noticed a fast onset of action of compounded analgesic cream; pain could be reduced within 20 minutes after the application of a phenytoin $10 \%$ cream [5].

Here we present a patient suffering from EMG documented CIAP and burning pain, tested for his response to phenytoin $10 \%$ cream in a single-blind fashion. Meanwhile, we use this single-blind response test always before we prescribe phenytoin cream, to rule out placebo-responders already during the first visit [6].

\section{Case presentation}

A man of 56 years old, started to have symptoms of restless legs many years ago, treated with pramipexol $0.125 \mathrm{mg}$ twice daily and temazepam $10 \mathrm{mg}$ once daily. He gave his informed consent for this case-presentation, as well as for a Youtube movie, where the patient explains what he noticed after the application of the phenytoin cream:

$$
\text { https://www.youtube.com/watch?v=9NHZ-B1iYII). }
$$

Since a year he experiences burning feet, cramps in leg muscles, and sensibility disturbances in both feet (walking on cotton). Pain scores on the 11-point NRS fluctuating between 6-9. He was referred to our clinic by the neurologist for further treatment of his burning pain. On examination, his DN-4 score was 5 , clearly indicative for a neuropathic pain.

Before prescribing a topical analgesic cream, we conducted a single-blind response test, applying placebo-cream on the right foot, and phenytoin $10 \%$ cream on the left foot (figure 1). Our instruction was: 'Here we have 2 different creams in a white tube, in both tubes we have mixed a number of compounds, and patients sometimes report to us that the cream in the tube reliefs the pain. Only in this tube (one of both) we are not sure about the mechanism of action, and in this other tube (one of both) we think we understand at least the mechanism of one of the compounds.' Within 20 minutes after application the patient clearly felt the difference: while the pain in the right foot remained more or less the same, pain in the left foot was reduced from a NRS score of 7 to 2 . This response led us to prescribe $10 \%$ compounded phenytoin cream. This cream we now develop together with a GMP certified pharmacist in the Netherlands.

\section{Burning Pain Reduction after Phenytoin cream}

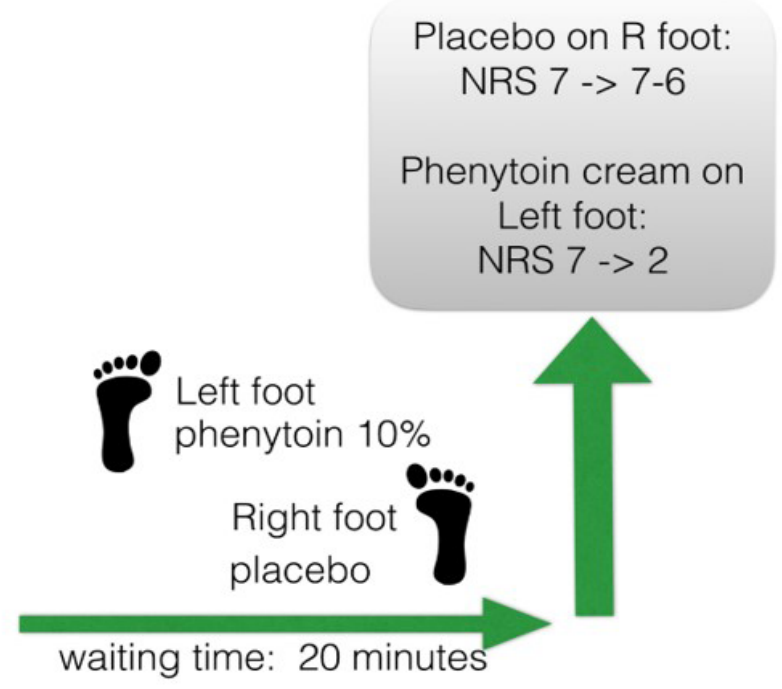

Discussion

Peripheral neuropathic pain is a complication of many polyneuropathies. We have developed a $10 \%$ compounded phenytoin cream, and in order to identify responders, we use a single-blind response test. During our first visit we apply placebo cream on one foot and phenytoin cream on the other foot, in cases where pain distribution and severity are comparable at both sides. For CIAP this is nearly always the case. In the case described above, the patient could correctly identify the active cream within a time-frame of 20 minutes.

Since our first introduction of the cream, we have treated more than 100 patients, most of those successfully, and we reported a series of 12 patients recently, based on a single-blind response test. [3] In these patients, phenytoin cream was significantly better compared to placebo. In order to further evaluate the cream in CIAP patients we started preparing a full powered RCT in CIAP patients, together with colleagues of the Medical Academic Centre in Utrecht. This study will provide further answers of the value of phenytoin for CIAP patients, and will analyze the contribution of the response-test to identify responders.

\section{Conflict of Interest}

The author is patent holder of two patents related to the topical formulations of phenytoin in the treatment of pain: 1) Topical phenytoin for the use in the treatment of peripheral neuropathic pain and 2) Topical pharmaceutical composition containing phenytoin and a (co-) analgesic for the treatment of chronic pain.

\section{References}

1. Warendorf J, Vrancken AF, van Schaik IN, Hughes RA, Notermans NC. Drug therapy for chronic idiopathic axonal polyneuropathy. Cochrane Database Syst Rev. 2017: 20; 6: CD003456.

2. Keppel Hesselink JM. Chronic idiopathic axonal neuropathy and pain, treated with the endogenous lipid mediator palmitoylethanolamide: A case collection. Int Med Case Rep J. 2013; 6: 4953.

3. Kopsky DJ, Keppel Hesselink JM. Phenytoin Cream for the Treatment of Neuropathic Pain: Case Series. Pharmaceuticals (Basel). 2018; 11: E53.

4. Keppel Hesselink JM, Notermans NC. Topical phenytoin formulations for pain in small fiber neuropathy, a pathogenetic approach. Gen Int Med Clin Innov. 2018; 3: 2-4.

5. Keppel Hesselink JM, Kopsky DJ. Topical phenytoin cream in small fiber neuropathic pain: Fast onset of perceptible pain relief. Int J Pain Relief. 2017; 1: 15-19.

6. Keppel Hesselink JM. Single-blind placebo-controlled response on phenytoin $10 \%$ in painful diabetic neuropathy. Gen Med Open. 2018; 2: 2-3. 